\title{
Assessment of Health-Related Quality of Life: The Cinderella of Peritoneal Dialysis?
}

\author{
Mercedes Moreiras-Plaza, Raquel Blanco-García, Cinthya Raquel Cossio-Aranibar, \\ and Gloria Rodriguez-Goyanes
}

Servicio de Nefrología, Complexo Hospitalario Universitario de Vigo, Pizarro 22, 36204 Vigo, Spain

Correspondence should be addressed to Mercedes Moreiras-Plaza, mercedes.moreiras.plaza@sergas.es

Received 6 February 2011; Accepted 6 May 2011

Academic Editor: Hulya Taskapan

Copyright ( $) 2011$ Mercedes Moreiras-Plaza et al. This is an open access article distributed under the Creative Commons Attribution License, which permits unrestricted use, distribution, and reproduction in any medium, provided the original work is properly cited.

\begin{abstract}
The studies of quality of life (QoL) are becoming increasingly interesting in clinical setting because their findings have implications for making decisions on resource allocation and health policies. The assessment of health-related QoL is especially directed to patients with chronic illnesses that cause progressive deterioration and limitations, and consume the bulk of financial resources for health. Among these chronic kidney disease and, more specifically the renal replacement therapy, is an important condition. Due to the diagnostic and therapeutic advances, we see a gradual increase both in the number of the dialysis population and its age, which leads to a growing interest in the study of these patients' QoL, as evidenced by more than one thousand articles published on this subject.
\end{abstract}

\section{Definition of Quality of Life}

Quality of life is a difficult variable to define. We distinguish two basic characteristics in the concept of QoL: subjectivity and multidimensionality. Subjectivity should be distinguished because the QoL is a unique perception for each individual, which reflects the patient's self-assessment about their own health, defined by medical and nonmedical aspects of their lives. In fact, patients use the possible negative impact or treatment side effects for the evaluation of any medical intervention. Therefore, the success or failure of a medical intervention is the QoL acquired or recovered after it, in spite of success or technical failure of the procedure itself. Moreover, QoL is a multidimensional concept that comprised not only aspects directly related to health but also other nonmedical aspects, but the autonomy, the retention of employment, the impact on family relationships, the economic resources, and many other life circumstances are connected as well.

Many definitions have been proposed for an individualized, dynamic, and multifaceted concept. Thus, it has been defined as "the value assigned by an individual per year of life, modified by the social disadvantage, the perception, the functional status, or deficiencies due to illness, treatment or accident", while the WHO definies it as: "the perception that individual makes about his position in life, within its cultural context and value system, and related to its goals and vital objectives." Perhaps one of the rough and clear definitions referred to QoL is "the measure resulted from the physical, mental, and social well-being, such as is perceived by each individual." The medical and nonmedical factors are both related: the illness, as well as having an impact on the physical area of a person, has impact on the personal psychological state and his social relations. So we use the term health related quality of life, which could have been formally defined as "The extent to which one's usual or expected physical, emotional and social well-being is affected by a medical condition or its treatment." The concept of health relatedquality of life (HRQOL) covers the impact of the disease or medical actions on the physical symptoms, functional status, and mental and social functioning [1-3]. 


\section{Quality of Life and Chronic Kidney Disease (CKD)}

Chronic kidney disease (CKD) has a great impact on HRQOL [4-7]. Symptoms appear and induce substantial changes in lifestyle from the early stages of the disease to the substitution treatment such as hemodialysis (HD), peritoneal dialysis (PD), or renal transplantation (RT). CKD is invariably associated with decreased HRQOL, and there is a correlation between the magnitude of the effect on HRQOL and glomerular filtration rate. The most affected HRQOL areas are work and leisure, family life, and sleep and rest. Although an adequate treatment of patients during the predialysis stage may slow the progression of CKD and that is recognized as an important factor of morbidity and mortality (and therefore of HRQOL) of kidney patients, the start of dialysis treatment is the patients' turning point in their concept of quality of life. They go from a situation of "normal life" (often idealized) to a state of "mortal danger" or "life without health" that requires dialysis to stay alive. There are several aspects that affected patients will feel altered.

(i) The Well-Being. The repetition and frequency of dialysis procedure constantly reminds one of the disease, favouring self-analysis that exaggerates physiological phenomena. On the other hand, decreased sexual desire and activity (present in up to $50 \%$ of patients) are experienced as a sign of advanced age and interfere with relationships. Finally, dietary restrictions are perceived (and therefore often ignored) as the deprivation of the last "pleasure" that patients keep.

(ii) Body Image. Issues like skin colour, body odour, loss of urinary function, sexual impotence, internal arteriovenous fistula or peritoneal catheter presence, scars, or abdominal distension help patients to perceive their bodies negatively and with inferiority feeling. And these feelings will limit the social and family relationships, encouraging introversion.

(iii) Autonomy. The frequency of dialysis (either HD or exchange sessions in PD) interferes and limits the lifestyle of patients. On the other hand, inferiority feelings facilitate physical deterioration, passivity, neglect, and dependency, often enhanced by the good-intentioned help from patient's family and social environment.

(iv) The Mental Attitude. Anxiety is always in the background due to daily contact with the disease and the risk of death. And that anxiety leads to distress, somatisation, obsessive attitudes, depression, aggression, and so forth.

Today, due to scientific and medical advances, patients survive with problems that were deadly time ago, but this survival is often associated with various degrees of disability, causing dependence and a greater need of both medical and social care. The progressive aging of the dialysis population and greater comorbidity is a fact, and the close relationship between HRQOL and mortality score confirms the importance of including quality-of-life markers in the clinical management of patients. Moreover, now patients not only have an expectation to survive but also expect to achieve a certain level of well-being.

\section{Measuring Instruments for HRQOL Assessment}

As most of the components of HRQOL cannot be observed directly, to quantify this so broad and "confused" term we need measuring instruments such as HRQOL questionnaires. These instruments involve both subjective and objective measures. The subjective assessments involve any aspect of patients' health status that comes directly from the patient without interpretation of the response by a health care provider. The objective assessment of patient status is needed to evaluate the impact of health on quality of life and formulate clinical intervention strategies $[1,3,4,7]$. The dimensions usually measured by HRQOL questionnaires are

(i) physical function: mobility, self-care, and work,

(ii) emotional Function: well-being, satisfaction, depression, and anxiety,

(iii) social function: social support, family, and social relationships,

(iv) cognitive function: ability to reason, think, concentrate, and remember,

(v) other general or specific symptoms: sleep disturbance, sexual function, energy/vitality, pain, life satisfaction, body image, and so forth.

HRQOL questionnaires were designed and validated for specific populations and cultures, and therefore they need to be adapted to the language and culture in which it will be used, preserving the semantic content and thus leading to the equivalence between the two populations. This process of adaptation must be made according to scientific standards and has a complex but validated methodology. Therefore, the use of free translations of the original questionnaire is considered improper. It is often preferable to use a HRQOL questionnaire adapted to a new one, because in this way not only studies can be made to compare large groups, but also this use will be faster and cheaper.

HRQOL instruments are used for three main purposes: to discriminate between patients with better and worse HRQOL at one time, to assess how much the quality of life has changed between two different time points, and to predict future HRQOL from a current measurement. For accomplishing these purposes, the questionnaires must meet certain requirements to ensure valid and reliable clinical data $[1,3]$. These requirements are the following.

(i) Viability. The best questionnaire will be useless if its implementation is difficult, costly, and complex for both patient and health professionals. This is why aspects such as simplicity, brevity and clarity of the questions, and ease of completion and correction must be valued.

(ii) Reliability. It indicates the degree to which a measurement is free from random error and refers to the degree 


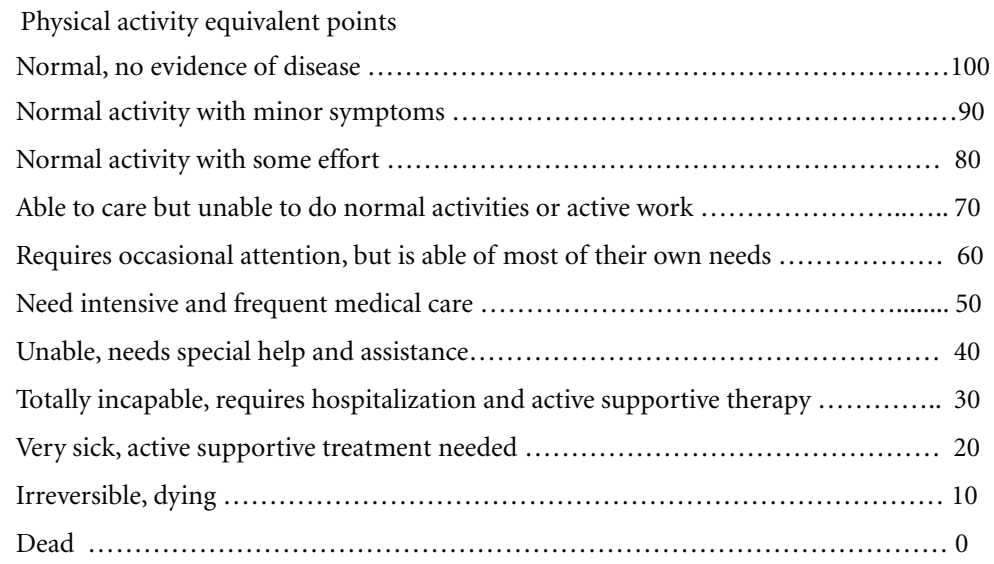

to which they can reproduce the results obtained under constant conditions, even in an extended series of repeated assessments. In addition, it refers to the homogeneity of items comprising the questionnaire.

(iii) Coverage. The questionnaire should include all basic dimensions that are important to members of the patient population and susceptible to being affected, positively or negatively, by interventions.

(iv) Validity. It measures only what they claim to measure.

(v) Sensitivity to Change. It shows the ability to detect and reflect true changes or differences in patient health.

(vi) Clinical Significance. The definition of "minimal clinical important difference", which is the rate of change in score of HRQOL questionnaire that should make the medical treatment, will be implanted.

(vii) Reproducibility. It shows stability over time, with little variation between intra- and interobservations.

(viii) Internal Consistency. All questions in one dimension (physical, emotional, etc.) measure the same concept.

The HRQOL questionnaires can be generic or diseasespecific. Generics questionnaires can be broadly used in different diseases and patient groups because they cover a wide range of dimensions of HRQOL, while the diseasespecific questionnaires are designed for a population or a disease including the most relevant dimensions for patients affected by a particular condition. Disease-specific instruments are more sensitive to clinical changes but do not allow HRQOL comparisons among patients with different pathologies. The most comprehensive assessment of HRQOL includes an assessment of both generic and disease-specific questionnaires [8].

\section{HRQOL Measurement Instruments in Nephrology}

The Generic questionnaires most commonly used in nephrology are the following.

(i) The Short Form-36 (SF-36). It is a short form of HRQoL scoring system with 36 items forming eight multi-item scale measuring physical functioning, role of physical, bodily pain, general health, vitality, social functioning, and role of emotions and emotional health $[7,9]$. It is one of the most commonly used HRQOL questionnaires used in CKD patients with or without maintenance dialysis therapy.

(ii) Nottinghan Health Profile. It consists of 38 items to measur 6 dimensions and is widely used because of its simplicity [10].

(iii) Sickness Impact Profile (SIP). It comprises 136 items to measure 12 dimensions [11].

(iv) The Karnofsky Scale. It was initially designed for cancer patients and is an ordinal scale ranging from a score 100 (normal state) to 0 (dead) which focuses exclusively on physical functioning and on role of limitations imposed by physical health [12] (see Figure 1).

(v) COOP-WONKA Charts [13] (see Figure 2).

(vi) The Squedule for Evaluation of Individual Quality of Life (SEIQOL). It is an established instrument that seeks to asses QoL on the basis of the domains that patients feel important $[5,7]$.

The specific questionnaires mostly used for kidney patients are the following.

(i) Kidney Disease Quality of Life Questionnaire (KDQOL). It includes the SF-36 as the generic core, supplemented with multi-item scales targeted at particular concern of patients with CKD and on dialysis [7]. 
Physical fitness

During the past 4 weeks

What was the hardest physical activity you could do for at least 2 minutes?

\begin{tabular}{l}
\hline $\begin{array}{l}\text { Very heavy (for example) } \\
\text { - Run, at a fast pace } \\
\text { - Carry a heavy load upstairs or uphill } \\
\text { - Jog, at a slow pace } \\
\text { - Climb stairs or a hill moderate pace }\end{array}$ \\
$\begin{array}{l}\text { Moderate (for example) } \\
\text { - Walk, medium pace } \\
\text { - Carry light load level ground }\end{array}$ \\
\hline $\begin{array}{l}\text { Light (for example) } \\
\text { - Walk, medium pace } \\
\text { - Carry light load level ground }\end{array}$ \\
\hline $\begin{array}{l}\text {-Wery light (for example) } \\
\text { - Walk, at a slow pace or not able to walk dishes }\end{array}$
\end{tabular}

(a)

Daily activities

How difficult has it been for you to do of your usual activities or tasks, both inside and outside the house because of your physical and emotional health?

\begin{tabular}{|l|l|}
\hline No difficulty at all & \\
\hline A little bit of difficulty & \\
\hline Some difficulty & \\
\hline Much difficulty & \\
\hline Could not do & \\
\hline
\end{tabular}

(c)
Feelings

During the past 4 weeks

To what extent have you been bothered by emotional problems such as feeling anxious, depressed, irritable or downhearted and sad?

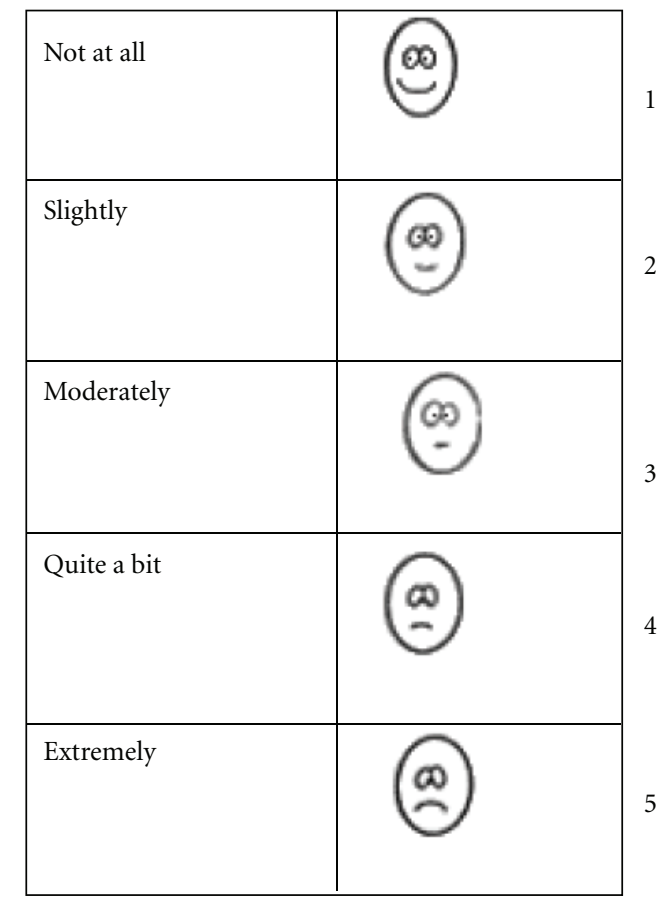

(b)

Social activities

During the past 4 weeks

Has your physical and emotional health limited your social activities with family, friends, neighbors, or groups?

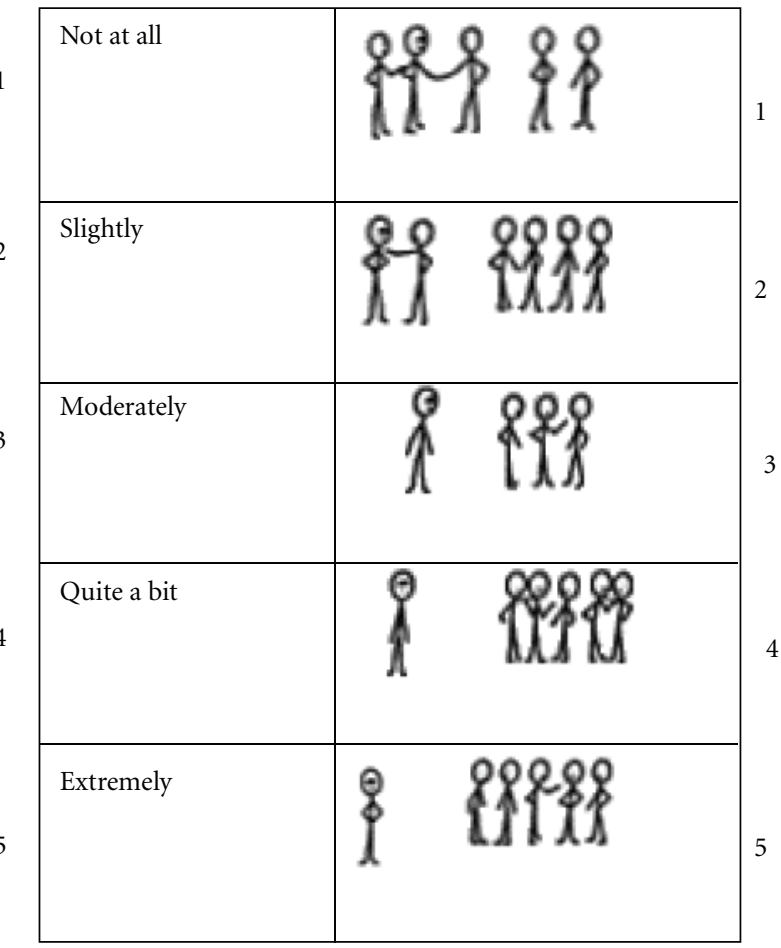

(d)

Figure 2: Continued. 
Change in health

How would you rate your overall health now compared to 4 weeks ago?

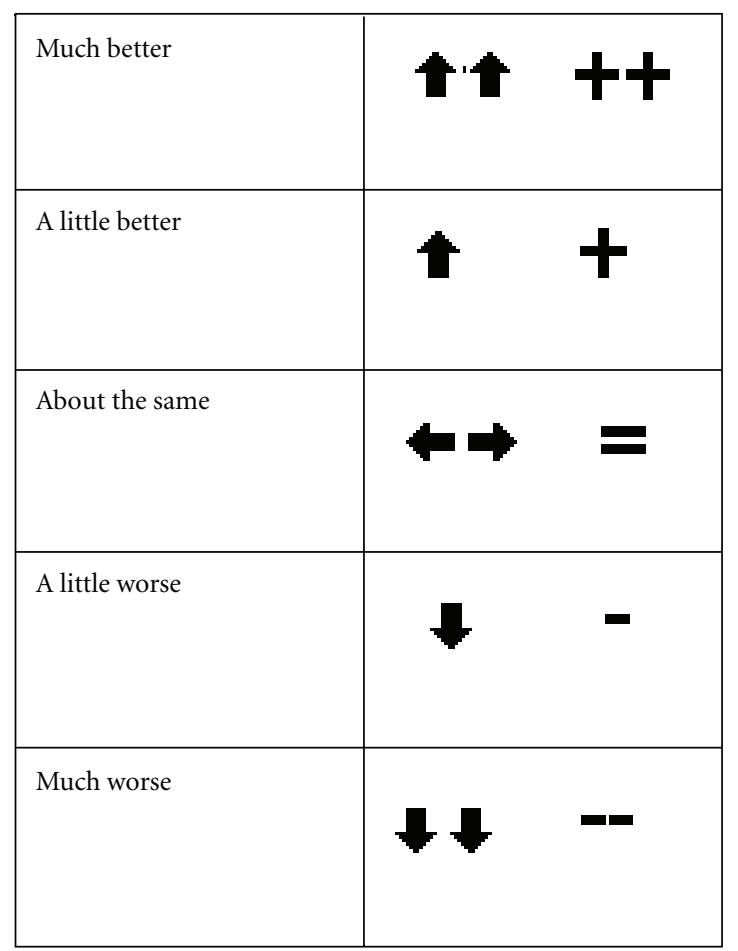

(e)
Overall health

During the past 4 weeks

How would you rate your health in general?

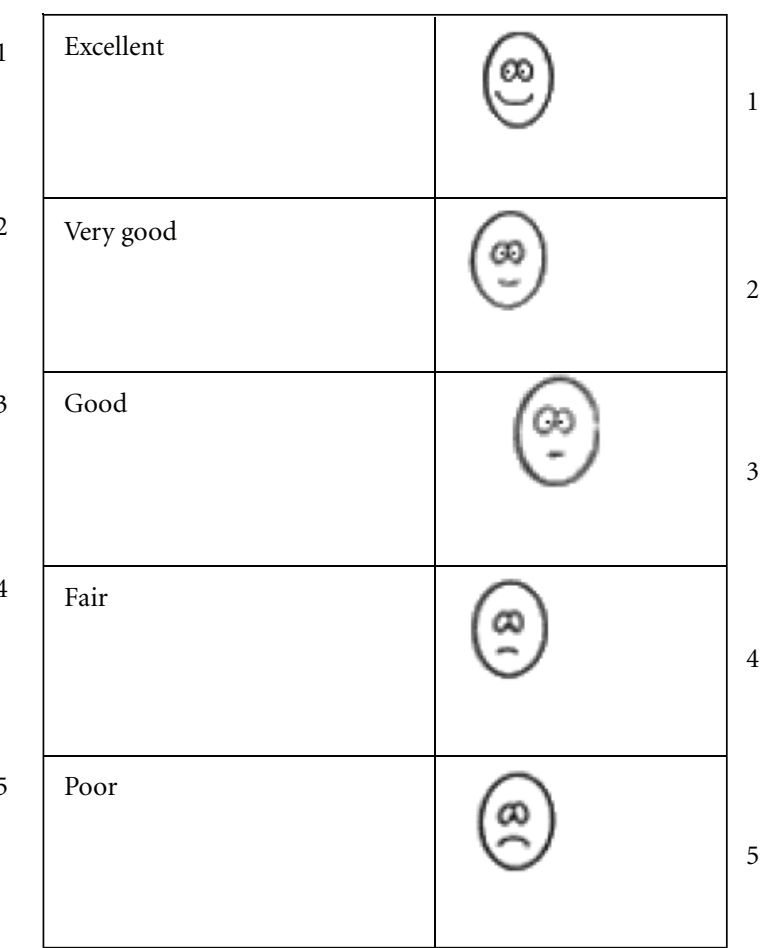

(f)

Pain

During the past 4 weeks

How much bodily pain have you generally had?

During the past 4 weeks

Was someone available to help you if you needed and wanted help? For example if you

- felt very nervous, lonely, or blue

- got sick and had to stay in bed

- needed someone to talk to

- needed help with daily chores

- needed help just taking care of yourself

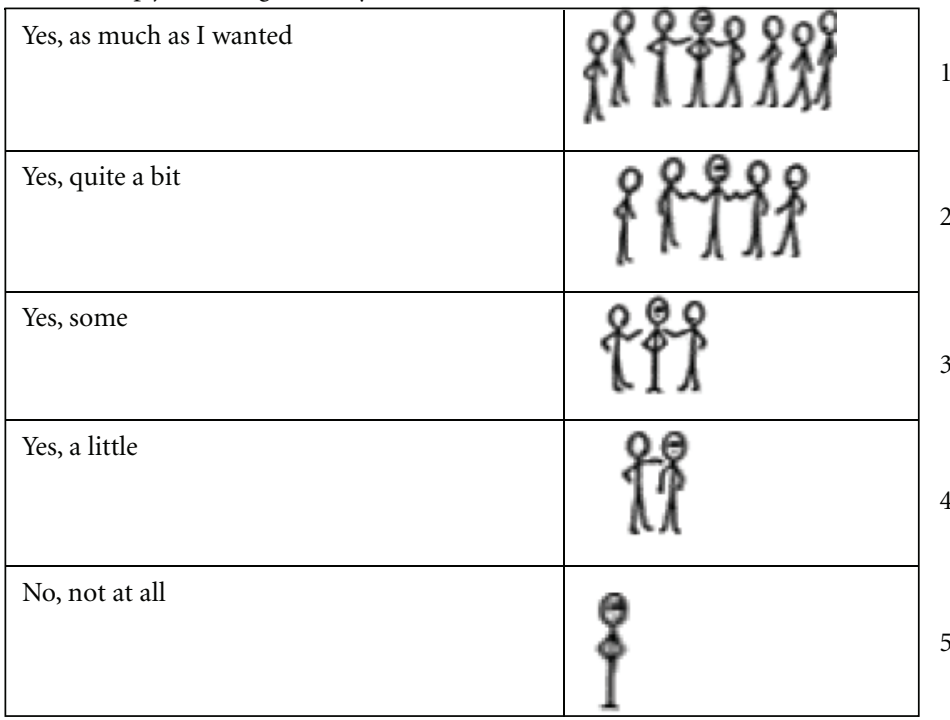

(g)

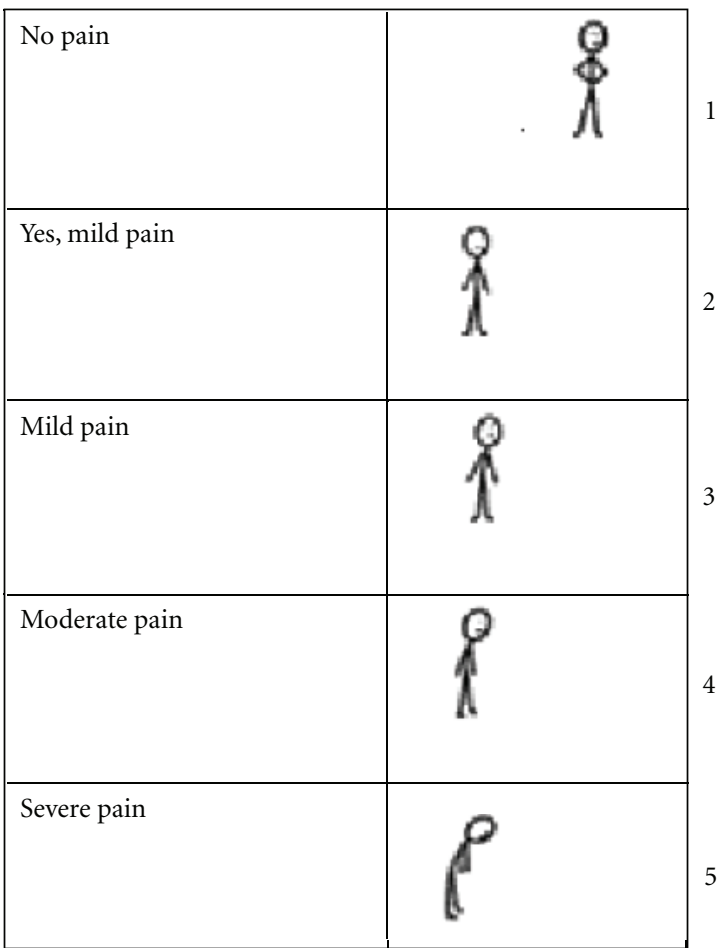

(h)

Figure 2: Continued. 


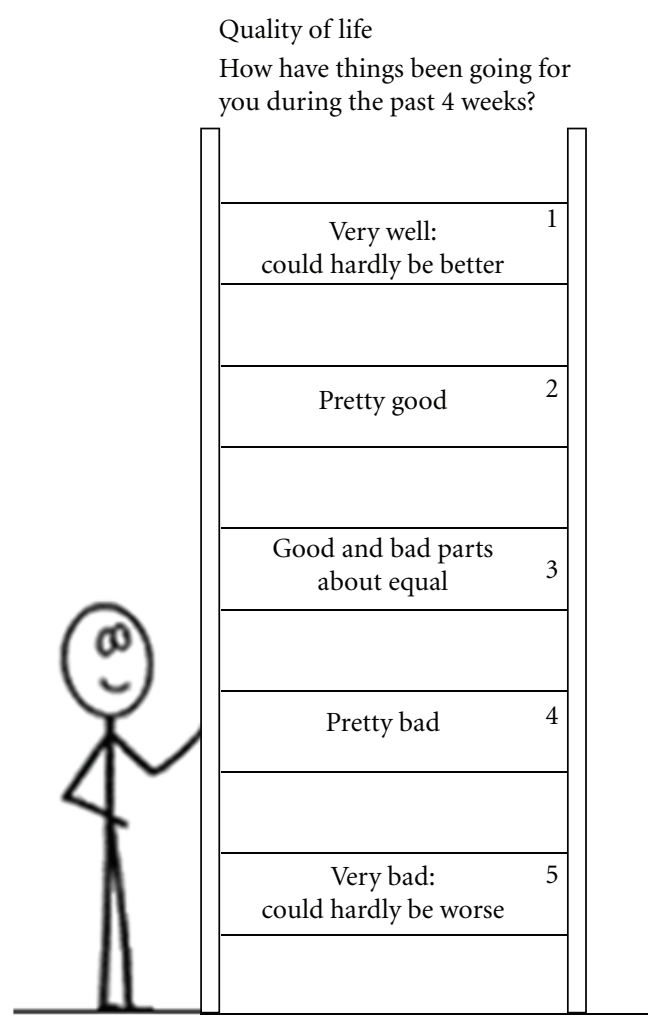

(i)

Figure 2: Example of COOP/WONCA charts.

(ii) KDQOL-SF. It is a short form of KDQOL that consists of the SF-36 plus a small set of 43 kidney-disease-targeted items. It is the most commonly used.

(iii) Parfrey Test. It is a Specific test for patients on hemodialysis and consists of 24 items distributed in two dimensions (physical and emotional) [14].

There is not a specific questionnaire for patients on peritoneal dialysis.

\section{HRQOL Studies and CKD}

Early HRQoL studies conducted in CKD were published for more than 20 years. Initially served to analyze the quality of life of uremic patients compared with the general population, showing in general lower scores in patients with CKD. The following studies focused on comparing patients on dialysis (any modality) and renal transplant, confirming a better HRQOL in the last, coming to score similar as to that of healthy populations. Most recent studies focus on the hemodialysis population (largest) and those technique and treatment factors that may influence morbidity and mortality of patients.

So, KDQOL-SF has been used in HEMO and DOPPS studies $[15,16]$. The HEMO study is randomized multicenter prospective one, which studies the effect of CKD and hemodialysis treatment on quality of life. The DOPPS (Dialysis Outcomes and Practice Patterns Study) is a prospective, multicenter study over a population of 10,000 patients that found an independent relationship between the physical dimension score of the SF-36 and hospitalization and mortality; this relationship was not shown in the mental dimension. The opposite results, however, were observed in the Spanish multicenter study, CALDIVIA, in which the mental dimension was the score that predicted hospitalization and mortality. The CHOICE Health Experience Questionnaire, which has 5 ESRD-specific domains (diet, freedom, body image, dialysis access, and symptoms) added to 8 generic domains in the SF-36 and 8 additional generic domains, is an instrument for measuring heath-related quality of life for patients with ESRD designed to evaluate the effectiveness of alternative dialysis modalities and prescriptions [17].

In general, most of HRQoL studies are observational and do not appreciate the change in HRQOL scores after interventions to improve health status.

\section{HRQOL Studies and Peritoneal Dialysis}

There are few, and most of them are comparative with haemodialysis, so many of the differences between the two populations reflect different policies for inclusion of patients on peritoneal dialysis [18-21]. Patients who are on peritoneal dialysis for the first time and by own choice tend to have better clinical state (physical, emotional, autonomous, social) maintain longer residual clearance, with the clinical implications that it supposes. Fewer jobs compare manual 
and automated peritoneal dialysis. Although a priori the automated mode (DPA) should have better HRQOL scores both for the technical characteristics and for the patient's autonomy, this has not been confirmed in recent reviews in which automated peritoneal dialysis has not been shown to provide a relevant improvement in HRQOL compared to manual mode, except in a greater availability of free time [22-25].

Among the studies comparing different modalities of dialysis therapy, we highlight the NECOSAD Study Group's [20] that analyzes the effect of starting dialysis with haemodialysis or peritoneal dialysis modalities on survival adjusted for quality of life, the meta-analysis of Cameron et al. [21] that studies HRQOL of patients undergoing different types of renal replacement therapy and the DiazBuxo's [19] report analyzing quality of life in hemodialysis and peritoneal dialysis patients.

Comparing the two types of peritoneal dialysis, we can outline the Van Biesen et al.'s [22] serie and the recent one of Michels et al. that did not find significant differences between the HRQOL in manual or automated peritoneal dialysis, as will as the De Wit et al.'s [23] report which shows only a slight improvement in mental health in patients on automated peritoneal dialysis and the Bro's study in which patients on APD had improved social and family issues and worse scores in the section on sleep.

\section{Practical Applications of the Study of HRQOL for PD Patients}

From the various studies about HRQoL conducted in different populations of kidney patients, factors with a proven to relationship and affecting the quality of life were identified. Thus, it is shown that the improvement of various parameters of the quality of life of patients was achieved with a better hematocrit. Other factors whose relationship has been confirmed are the predialysis control, the tolerance to dialysis procedure, the psychological situation, and finally sex and age. Treatment of depression, pain, and sleep disorders and changes in the regimen of dialysis therapy have been suggested to have positive effects on HRQOL.

It is obvious that it would be much easier for everyone to translate the results of therapeutic measures of HRQOL to specific therapeutic manoeuvres, but unfortunately, and due to the peculiarities of the concept of quality of life, that is not possible. Many times, the subjective factors, derived or not from actual physical situations, determine the self-concept of quality of life of patients. That is why the results of the questionnaires should be analyzed carefully and thoroughly, dedicating to the discussion with the patient the necessary time to carry out actions aimed at improving the deficient aspects. A recent paper focuses on this subject suggesting that these tests could be applied as a nurse-led case management programme [26].

The measurable parameters (hematocrit, KTV, albumin, etc.) are not always the only ones on which we must depend, and responses to questionnaires of HRQOL can focus on what is more important for the patients. Here are some simple measures applicable to peritoneal dialysis that can benefit many aspects assessed with HRQOL questionnaires.

(i) Body Image. A lower insertion of a shorter peritoneal catheter makes it easily concealable even under swimwear; reducing the exchange volume as much as adequacy permits; avoiding the premature loss of residual renal function; giving instructions about skin care and oral hygiene.

(ii) Physical State. Controlling the anaemic status, the nutrition, and the possible associated diseases (digestive, vascular, cardiac); starting dialysis sequentially.

(iii) Work and Social Life. Accommodating as much as possible the schedules of peritoneal exchanges to the usual scheme of patient's life; offering automated peritoneal dialysis; facilitating alternative schedules of treatment or guidelines for the various social contingencies that may arise.

(iv) Mobility and Hospital Dependence. Minimizing hospitalization, spacing periodic controls (telemedicine), facilitating telephone contact, and cooperating with home care services.

(v) The Self-Care. Avoiding overload the patients with a "hospitalary method" for their daily care that will constantly remind them of their illness, but without letting them feel abandoned; seeking alternatives to a possible lack of infrastructure to perform the technique: involving the family only just to discourage the feeling of dependency or disability; using the minimum number of exchanges that adequacy permits.

(vi) The Emotional State. Maintaining a fluid dialogue between caregiver and patient; discussing all aspects of nephrology treatment; resolving doubts and problems, looking for extrarenal situations that may interfere with the perception of well-being; stopping the progress of any depressive state.

(vii) Diet. Reducing as much as possible the diet restrictions ("a little bit of everything, a lot of anything") and making that patient see the reason for the limitations indicated; giving menu suggestions, dietary supplements where necessary, or oral moisturizers to reduce the thirst sensation.

(viii) Sex, Sleep, and Pain. Asking patients directly and openly about these issues and trying to alleviate them (and not considering them as "inevitable disease's consequences").

\section{Final Comments}

After almost 20 years from implementation, HRQOL questionnaires are not used routinely, perhaps because its usefulness is not known, not only in the field of research (analysis after pharmacological or therapeutic interventions, cost-effectiveness assessment of new technologies) but also in the clinical setting (descriptive analysis of population, like 
a parameter of clinical quality). K-DOQI Guidelines 2000 recommend, in form of opinion, the periodic assessment of HRQOL of patients in any of the stages of CKD and in the specific population on peritoneal dialysis. Instruments recommended are the SF-36, KDQOL, and COOP-WOKA sheets, advising to always use the same one to improve data analysis over time.

For practical purposes, and while experience is not acquired in handling and interpretation, the Karnofsky scale and CCOP-WONCA sheets are easily applicable instruments: the time spent in filling them is little, they are attractive to patients and easy to evaluate, and their use lights clinical aspects that otherwise would be ignored.

\section{References}

[1] M. A. Testa and D. C. Simonson, "Assessment of quality-of-life outcomes," The New England Journal of Medicine, vol. 334, no. 13, pp. 835-840, 1996.

[2] World Health Organization, Quality of Life Assessment. An Annotated Bibliography, WHO (MNIH/PSF/94.1), Geneva, Switzerland, 1994.

[3] P. Rebollo and F. B. Ortega, "New trends on health related quality of life assessment in end-stage renal disease patients," International Urology and Nephrology, vol. 33, no. 1, pp. 195202, 2002.

[4] F. O. Finkelstein, D. Wuerth, and S. H. Finkelstein, "Health related quality of life and the CKD patient: challenges for the nephrology community," Kidney International, vol. 76, no. 9, pp. 946-952, 2009.

[5] K. Abdel-Kader, L. Myaskovsky, I. Karpov et al., "Individual quality of life in chronic kidney disease: influence of age and dialysis modality," Clinical Journal of the American Society of Nephrology, vol. 4, no. 4, pp. 711-718, 2009.

[6] M. S. Thong, A. A. Kaptein, Y. Benyamini et al., "Netherlands Cooperative Association between a self-rated health question and mortality in young and old dialysis patients: a cohort study," The American Journal of Kidney Diseases, vol. 52, p. 111, 2008.

[7] K. Kalantar-Zadeh and M. Unruh, "Health related quality of life in patients with chronic kidney disease," International Urology and Nephrology, vol. 37, no. 2, pp. 367-378, 2005.

[8] T. V. Perneger, M. Leski, C. Chopard-Stoermann, and P. Y. Martin, "Assessment of health status in chronic hemodialysis patients," Journal of Nephrology, vol. 16, no. 2, pp. 252-259, 2003.

[9] E. G. Lowrie, R. B. Curtin, N. LePain, and D. Schatell, "Medical outcomes study short form-36: a consistend and powerful predictor of morbidity and mortality in dialysis patients," The American Journal of Kidney Diseases, vol. 41, no. 6, pp. 12861292, 2003.

[10] S. M. Hunt, S. P. McKenna, and J. McEwen, "A quantitative approach to perceived health status: a validation study," Journal of Epidemiology and Community Health, vol. 34, no. 4, pp. 281-286, 1980.

[11] M. Bergner, R. A. Bobbitt, W. B. Carter et al., "The sickness impact profile: development and final revision of a health status measure," Medical Care, vol. 19, no. 8, pp. 787-805, 1981.

[12] D. Karnofsky and J. Burchenel, "The clinical evaluation of chemotherapeutic agents in cancer," in Evaluation of Chemotherapeutiic Agents, C. Maclead, Ed., pp. 191-205, Columbia University Press, New York, NY, USA, 1949.
[13] E. C. Nelson, J. H. Wasson, J. Kirk et al., "Assessment of function in routine clinical practice: description of the COOP Chart method and preliminary findings," Journal of Chronic Diseases, vol. 40, pp. S55-S63, 1987.

[14] P. S. Parfrey, H. Vasavour, M. Bullock et al., "Development of a health questionnaire specific for end-stage renal disease," Nephron, vol. 52, no. 1, pp. 20-28, 1989.

[15] M. Unruh, R. Benz, T. Greene et al., "Effects of hemodialysis dose and membrane flux on health-related quality of life in the HEMO study," Kidney International, vol. 66, no. 1, pp. 355366, 2004.

[16] D. L. Mapes, A. A. Lopes, S. Satayathum et al., "Health-related quality of life as a predictor of mortality and hospitalization: the Dialysis Outcomes Practice Patterns Study (DOPPS)," Kidney International, vol. 64, no. 1, pp. 339-349, 2003.

[17] A. W. Wu, N. E. Fink, K. A. Cagney et al., "Developing a health-related quality-of-life measure for end-stage renal disease: the CHOICE health experience questionnaire," The American Journal of Kidney Diseases, vol. 37, no. 1, pp. 11-21, 2001.

[18] R. Gokal, M. Figueras, A. Ollé et al., "Outcomes in peritoneal dialysis and hemodialysis: a comparative assessment of survival and quality of life," Nephrology Dialysis Transplantation, vol. 14, supplement 6, pp. 24-30, 1999.

[19] J. A. Diaz-Buxo, E. G. Lowrie, N. L. Lew et al., "Quality-of-life evaluation using short form 36: comparison in hemodialysis and peritoneal dialysis patients," The American Journal of Kidney Diseases, vol. 35, no. 2, pp. 293-300, 2000.

[20] NECOSAD Study Group, "Effect of starting dialysis with hemodialysis or peritoneal dialysis on survival adjusted for quality of life," Kidney International, vol. 64, pp. 2222-2228, 2003.

[21] J. I. Cameron, C. Whiteside, J. Katz, and G. Devins, "Differences in quality of life across renal replacement therapies: a meta-analytic comparison," The American Journal of Kidney Diseases, vol. 35, no. 4, pp. 629-637, 2000.

[22] W. Van Biesen, N. Veys, R. Vanholder, and N. Lameire, “The role of APD in the improvement of outcomes in an ESRD program," Seminars in Dialysis, vol. 15, no. 6, pp. 422-426, 2002.

[23] G. A. De Wit, M. P. Merkus, R. T. Krediet, and F. T. De Charro, "A comparison of quality of life of patients on automated and continuous ambulatory peritoneal dialysis," Peritoneal Dialysis International, vol. 21, no. 3, pp. 306-312, 2001.

[24] S. Bro, J. B. Bjorner, P. Tofte-Jensen et al., "A prospective, randomized multicenter study comparing APD and CAPD treatment," Peritoneal Dialysis International, vol. 19, no. 6, pp. 526-533, 1999.

[25] W. M. Michels, S. van Dijk, M. Verduijn et al., "Quality of life in automated and continuous ambulatory peritoneal dialysis," Peritoneal Dialysis International, vol. 31, pp. 138-147, 2011.

[26] S. K. Chow and F. K. Y. Wong, "Health-related quality of life in patients undergoing peritoneal dialysis: effects of a nurse-led case management programme," Journal of Advanced Nursing, vol. 66, no. 8, pp. 1780-1792, 2010. 


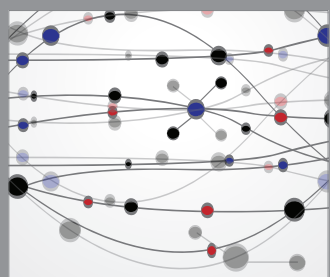

The Scientific World Journal
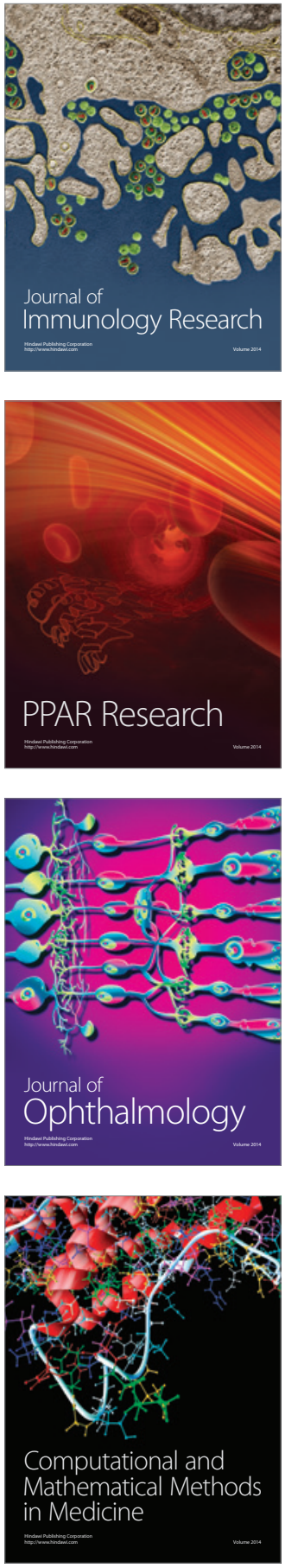

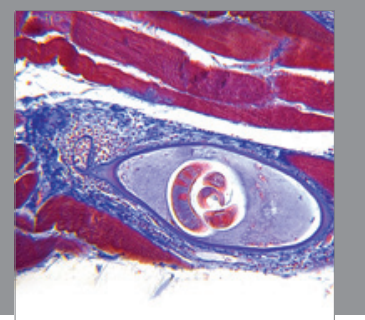

Gastroenterology

Research and Practice
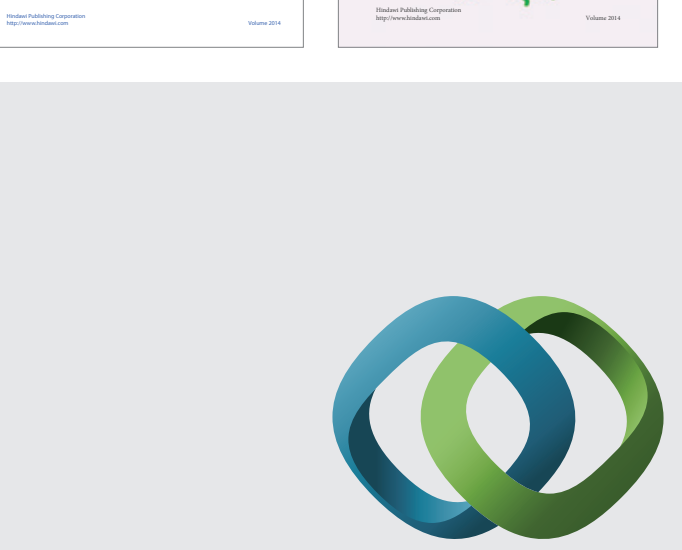

\section{Hindawi}

Submit your manuscripts at

http://www.hindawi.com
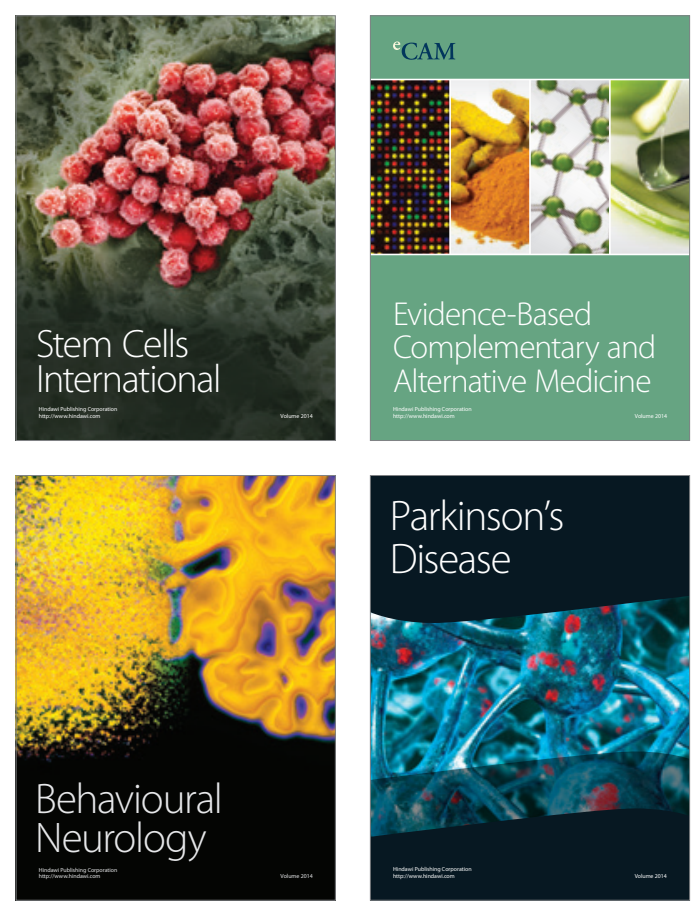

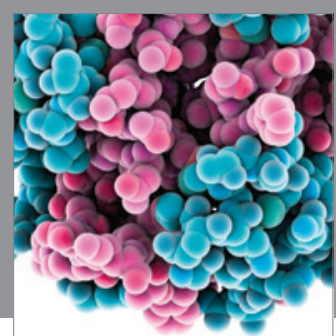

Journal of
Diabetes Research

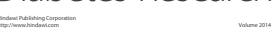

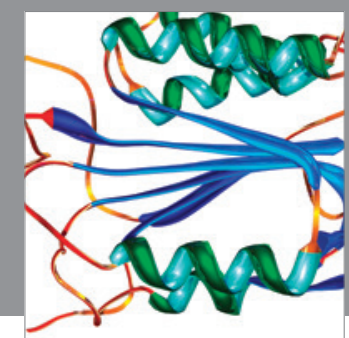

Disease Markers
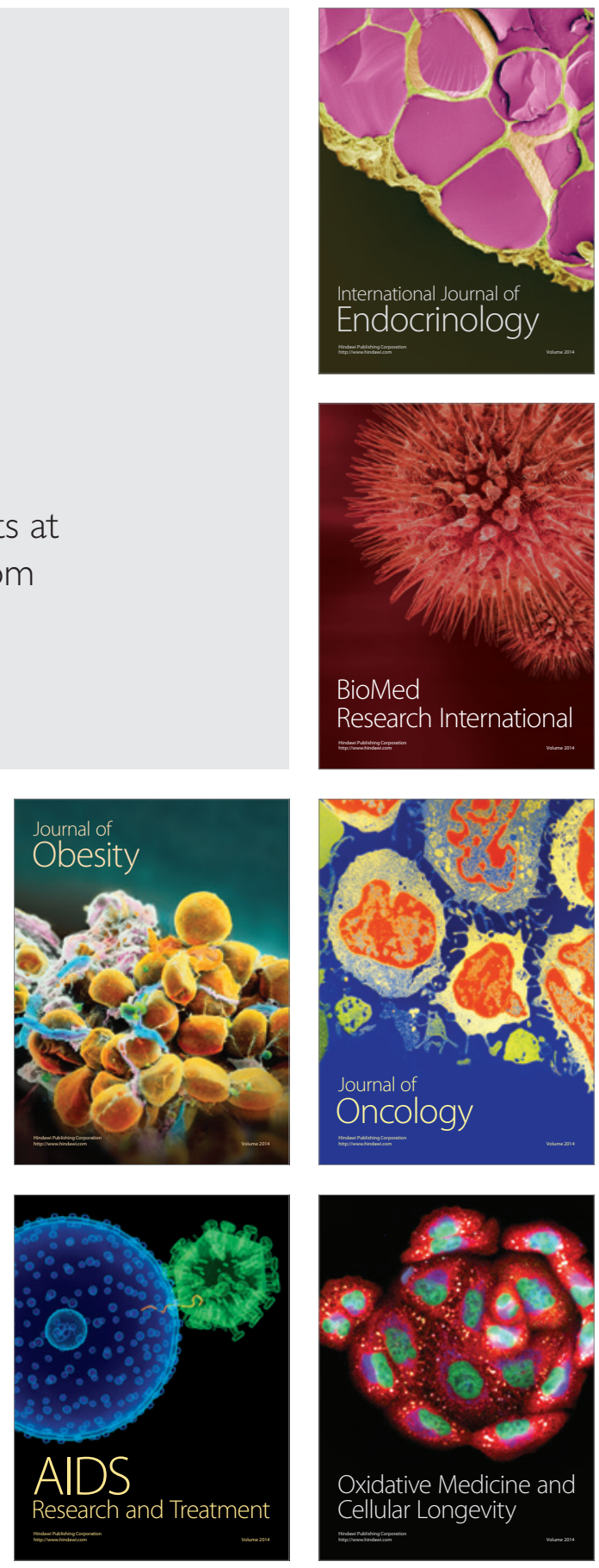